\title{
Equilibrium Measures on Finite Networks: Effective Resistance and Hitting Time
}

\author{
Enrique Bendito, Angeles Carmona and Andrés M. Encinas \\ Departament de Matemàtica Aplicada III \\ Universitat Politècnica de Catalunya. España
}

We aim here at showing how the equilibrium measures for a finite network can be used to obtain simple expressions for both Green and Poisson kernels and hence we can deduce nice expressions of the hitting time and the effective resistance. Also we will give a new and simple proof, using equilibrium measures, of the algorithm that compute the resistive inverse of a effective resistances matrix. It is well known, in the context of finite network, that the effective resistance between two nodes can be obtained from the solution of certain Dirichlet problem and hence from its Green function ([4]), and the same happens for the hitting time $([2,5]$.) On the other hand, Tetali [8] gives an expression of the hitting time in terms of effective resistances and Coppersmith et al. [3], Metz [6] and Ponzio [7] give an expression of the Green function in terms of effective resistances by using different techniques. So one can think that all the above mentioned concept have a strong relation. We believe that this relation can be explained throughout equilibrium measures.

Let $\Gamma=(V, E, c)$ be a finite network, where $V$ is the vertex set, $E$ is the edge set and $c: V \times V \longrightarrow \mathbb{R}$ is the conductivity function of the network, i.e., $c(x, y)>0$ if $x \sim y, c(x, y)=0$ if $x \nsim y$. The Laplacian matrix of $\Gamma$ is the $n \times n$-matrix $\mathcal{L}=\mathcal{L}(\Gamma)$ indexed by the vertices of $\Gamma$, whose entries are given by $\mathcal{L}_{x y}=-c(x, y)$ and $\mathcal{L}_{x x}=c(x)=\sum_{y \in V} c(x, y)$.

The authors have proved in previous works (see, for instance [1]) that, in the context of Potential Theory, the Laplacian, considered as a kernel, verifies the equilibrium principle, i.e.: for every proper set $F \subset V$ there exists $\mu \in \mathcal{M}^{+}(F)$ such that

$$
\begin{aligned}
& \mathcal{L} \mu=1 \quad \text { in } F, \\
& \mathcal{L} \mu \leq 1 \quad \text { in } F^{c} .
\end{aligned}
$$

Moreover, the support of $\mu$ is $F$. The solution of this problem is called equilibrium measure for $F$. The existence of equilibrium measures will be crucial 
for that work in order to obtain simple expressions for the Green and Poisson kernels, the effective resistance and the hitting time. More precisely we will work with equilibrium measures for proper sets of the form $V-\{z\}$ for $z \in V$, which will be denoted by $\nu_{z}$ and called equilibrium measures of the network.

Now we consider the Green and Poisson kernels and we give their expressions in terms of the above equilibrium measures.

Let $F \subset V, \delta(F)$ its vertex boundary and $\bar{F}=F \cup \delta(F)$. A function $G$ : $\bar{F} \times F \longrightarrow \mathbb{R}$ is called the Green kernel for $F$ if $G_{y}(x)=G(x, y)$ is the solution of the following semi-homogeneous Dirichlet Problem:

$$
\begin{aligned}
\mathcal{L} G_{y}=\delta_{y} & \text { in } F, \\
G_{y}=0 & \text { in } \delta(F) .
\end{aligned}
$$

In addition, $G$ is a symmetric kernel.

A function $P: \bar{F} \times \delta(F) \longrightarrow \mathbb{R}$ is called the Poisson kernel for $F$ if $P_{y}(x)=$ $P(x, y)$ is the solution of the following semi-homogeneous Dirichlet Problem:

$$
\begin{array}{ll}
\mathcal{L} P_{y}=0 & \text { in } F \\
P_{y}=\delta_{y} & \text { in } \delta(F) .
\end{array}
$$

Then, the Green kernel for $V-\{z\}$ can be obtain in terms of equilibrium measures in the following way:

$$
G(x, y)=\frac{1}{n}\left(\nu_{z}(x)-\nu_{y}(x)+\nu_{y}(z)\right)
$$

And the Poisson kernel for $V-\{y, z\}$ can be expressed using equilibrium measures as:

$$
P(x, y)=\frac{\nu_{z}(x)-\nu_{y}(x)+\nu_{y}(z)}{\nu_{z}(y)+\nu_{y}(z)} \quad \text { and } \quad P(x, z)=\frac{\nu_{y}(x)-\nu_{z}(x)+\nu_{z}(y)}{\nu_{z}(y)+\nu_{y}(z)} \text {. }
$$

The effective resistance between any par of vertices $y$ and $z$ is defined as $R_{z y}=I(v)^{-1}$, where $v$ is the solution of the following Dirchlet problem:

$$
\begin{aligned}
& \mathcal{L} v=0 \quad \text { in } V-\{y, z\} \\
& v(y)=1 \\
& v(z)=0 .
\end{aligned}
$$


Using the Poisson kernel for $V-\{y, z\}$ we obtain

$$
R_{y z}=\frac{\nu_{z}(y)+\nu_{y}(z)}{n}
$$

On the other hand, the hitting time $H(x, y)$ from $x$ to $y$, defined as the expected number of steps for a reversible Markov chain before state $y$ is reached, when started from state $x$ verifies:

$$
\begin{aligned}
& \mathcal{L} H_{y}(x)=c(x), \quad x \in V-\{y\}, \\
& H_{y}(y)=0 .
\end{aligned}
$$

And hence using the expression of the Green kernel we obtain:

$$
H(x, y)=\frac{1}{n} \sum_{z \in V} c(z)\left(\nu_{z}(y)+\nu_{y}(x)-\nu_{z}(x)\right) .
$$

We can obtained straightforward formulas for the Green and Poisson kernels and the hitting time, in terms of effective resistance, simply replacing (1) in the their expressions:

$$
\begin{array}{ll}
G(x, y)=\frac{1}{2}\left(R_{x z}+R_{y z}-R_{x y}\right), & H(x, y)=\frac{1}{2} \sum_{z \in V} c(z)\left(R_{x y}+R_{y z}-R_{x z}\right), \\
P(x, y)=\frac{1}{2 R_{y z}}\left(R_{x z}+R_{y z}-R_{x y}\right), & P(x, z)=\frac{1}{2 R_{y z}}\left(R_{x y}+R_{y z}-R_{x z}\right) .
\end{array}
$$

\section{References}

[1] E. Bendito, A. Carmona and A. M. Encinas, Solving Boundary Value Problems on Networks Using Equilibrium Measures, J. Func. Anal., 171 (2000) 155-176.

[2] F.R.K. Chung and S.T. Yau, Discrete Green's functions, J. Comb. Theory, Ser. A, 91 (2000) 191-214.

[3] D. Coppersmith, P. Doyle, P. Raghavan and M. Snir, Random Walks on Weighted Graphs and Applications to On.line Algorithms, J. Assoc. Comput. Mach., 40 (1993) 421-453.

[4] P.G. Doyle and J.L. Snell, "Random Walks and Electric Networks," Math. Assoc. America, 1984.

[5] A. Grigor'yan and A. Telcs, Sub-Gaussian estimates of heat kernels on finite graphs, Duke Math. J., to appear. 
[6] V. Metz, Shorted operators: An application in Potential Theory, Linear Algebra Appl., 264 (1997) 439-455.

[7] S. Ponzio, The Combinatorics of Effective Resistances and Resistive Inverses, Inform. and Comput., 147 (1998) 209-223.

[8] P. Tetali, Random walks and the effective resistance of networks, Adv. Appl. Probab., 26 (1991) 820-824. 